\title{
2. La fragilité
}

(c) Springer-Verlag France 2010

\section{1 Éléments biologiques de la fragilité \\ C. Swine \\ Marie de Saint-Hubert (UCL/IRSS, Belgique)}

Se référant aux modèles physiopathologiques de la fragilité, avec l'exemple de la sarcopénie, les voies principalement concernée sont d'une part l'inflammation chronique et d'autre part la voie neuroendocrine. L'activation de ces voies est déterminée et modulée entre autres par le vieillissement cellulaire, la sénescence réplicative (télomères), le stress oxydatif, la réponse de prolifération des cellules $\mathrm{T}$, les lésions de l'ADN, les variations dans l'expression des gènes etc. Ces régulations deviennent paradoxalement moins complexes avec le vieillissement ce qui entraîne une plus grande ampleur et variabilité des réponses par défaut des mécanismes compensatoires. Ainsi, les composantes de la réaction immunitaire sont différemment affectées par le vieillissement, ce qui engendre un déséquilibre entre cytokines pro- et anti-inflammatoires, en faveur d'un état inflammatoire chronique de bas grade, dénommé inflamm-ageing (Franceschi et al., 2000a). Les taux de différents médiateurs inflammatoires augmentent avec l'âge, que ce soient l'IL-6 (Cohen et al., 2003 ; Bruunsgaard et al., 2003c), la CRP (Ballou et al., 1996), ou le TNF- $\alpha$ (Bruunsgaard et Pedersen, 2003c). Il existe également des associations transversales entre des marqueurs inflammatoires et le phénotype de fragilité décrit par Fried (Leng et al., 2002 ; Leng et al., 2007), ou des caractéristiques de la fragilité : vitesse de marche, grip strength, force musculaire (Taaffe et al., 2000 ; Cesari et al., 2004 ; Colbert et al., 2004) et les limitations fonctionnelles (Ravaglia et al., 2004). Les taux d'IGF-1 abaissés et d'IL-6 élevés agissait de façon synergique et sont significativement associés à une augmentation des incapacités physiques (Cappola et al., 2003). Concernant l'immunosénescence proprement dite, un phénotype à risque (immune risk profile, IRP) a été décrit consistant en une inversion du rapport lymphocytaire $\mathrm{T}$ (ratio $\mathrm{CD} 4+/ \mathrm{CD} 8+<1$ ) et une diminution de la prolifération lymphocytaire in vitro (Wikby et al., 1998).
La séropositivité pour le CMV (Pawelec et al., 2004) a également été associée à ce phénotype qui prédit une mortalité accrue (Wikby et al., 1998).

Il y a donc de nombreux candidats biologiques comme témoins ou biomarqueurs de ces modifications et dysfonctionnements, pour nous documenter sur les correspondants biologiques de la fragilité. Certains pourraient s'avérer utiles dans la pratique en complément des indicateurs cliniques pour identifier les risques liés à la fragilité et stratifier les niveaux de risque.

\section{2}

\section{À la recherche d'un paradigme pertinent} au plan clinique et au plan de la recherche H. Bergman

Affaires scientifiques, fonds de la recherche en santé du Québec, départements de médecine, de médecine de famille et d'oncologie, université McGill, hôpital général Juif, Montréal, Québec

« Je reconnais la fragilité à l'œil, mais je ne sais pas comment la définir », affirment couramment les cliniciens. Voilà un commentaire qui ne surprend pas car, malgré l'élargissement accru des connaissances - la documentation scientifique foisonne de modèles, de critères et de définitions -, il n'existe aucune définition de la fragilité qui soit largement admise. Des travaux réalisés jusqu'à maintenant ont ouvert de nouveaux horizons fort intéressants même si le consensus demeure un objectif à atteindre.

La présentation abordera les modèles de fragilité existants, la relation complexe entre les concepts de fragilité, de vieillissement et d'incapacité, ainsi que les implications du concept au plan clinique et au plan de la recherche. Éventuellement, les travaux sur la fragilité serviront aux cliniciens, aux personnes âgées et à la société dans la mesure où ils permettront de déterminer des interventions efficaces en promotion de la santé, en prévention, en réadaptation et dans le domaine du traitement et des soins. 